\title{
Sedation in the Intensive Care Unit
}

\author{
Valerie Page $^{1,2} \cdot$ Cathy McKenzie ${ }^{3,4}$
}

Accepted: 23 March 2021 / Published online: 24 April 2021

(C) Springer Science+Business Media, LLC, part of Springer Nature 2021

\begin{abstract}
Purpose of Review This narrative review illustrates literature over the last 5 years relating to sedation delivery to mechanically ventilated adult patients in intensive care units.

Recent Findings There has been an increase in dexmedetomidine-related publications but although systematic reviews suggest dexmedetomidine reduces delirium, agitation, and length of stay, clinical trials have not supported these findings. It is likely to be useful for the managing patients with persisting agitation. Guidelines continue to recommend lightly sedating patients but considerable variation remains in clinical practice and in research trials. Protocols with no sedative infusions and morphine boluses as needed are feasible and safe, while educational interventions can decrease sedation-related adverse events.

Summary Research trials have mainly focused on individual drugs rather than practice. Given evidence is slow to translate into practice; work is needed to understand and respond to the concerns of clinicians regarding deep sedation and agitation.
\end{abstract}

Keywords Sedation $\cdot$ Ventilation $\cdot$ Intensive care $\cdot$ Critical care $\cdot$ Dexmedetomidine $\cdot$ Protocol

\section{Introduction}

All evidence-based international guidelines regarding sedation for mechanically ventilated patients in intensive care are consistent in their recommendations. The US, South American and Iberian, German, and UK guidance all recommend minimal sedation [1-4]. The goal is a patient easily roused, comfortable with good pain control, unless deep sedation is clinically required.

Since 2016, sedation-related publications have been focused on determining the safety and efficacy of the sedative drugs in common use with an emphasis on dexmedetomidine, as well as the effectiveness of protocols or bundles. This

This article is part of the Topical Collection on Critical Care Anesthesia

Valerie Page

valerie.page2@nhs.net

1 Department of Anaesthesia, Watford General Hospital, Vicarage Road, Watford WD18 0HB, UK

2 Department of Surgery and Cancer, Imperial College London, London SW7 2AZ, UK

3 Faculty of Life Sciences and Medicine, Kings College London, London SE1 9RT, UK

4 Pharmacy and Critical Care, Kings College Hospital, London SE5 9RS, UK narrative review will outline some of the findings over the last five years relating to common sedative drugs and sedation delivery protocols.

\section{Search Strategy}

PubMed search 22nd Dec for the terms "sedation and intensive care", "sedation \& critical care", "sedation and mechanical ventilation", "sedation and delirium", "sedation and agitation", peer reviewed articles on humans in English published in last 5 years and separate search on 25th Jan for "sedation and COVID-19".

\section{Guidelines}

A number of guidelines or bundles of care were designed or updated to guide clinicians in consistent delivery of optimal sedation to mechanically ventilated critically ill patients.

The Society of Critical Care Medicine added Immobility (mobilization/rehabilitation) and Sleep (disruption) to their 2018 update of the 2013 Pain, Agitation, and Delirium guidelines including publications up to October 2015 [1•]. Rehabilitation/mobilization interventions were recommended to reduce ICU-acquired muscle weakness due to immobility 
with starting and stopping criteria for rehabilitation/mobility. Multicomponent protocols were recommended to promote sleep, including volume control ventilatory modes and nocturnal strategies to reduce noise and light, but no recommendations made regarding medications. These guidelines included 37 actionable recommendations and 2 best practice statements. Only two of the recommendations were strong, (i) using a neuropathic pain medication (e.g. gabapentin, carbamazepine, and pregabalin) with opioids for neuropathic pain management in critically ill patients and (ii) advising against volatile anaesthetic agents for procedural pain management.

Drs Pisani, Devlin, and Skrobik published a comprehensive exploration of the evidence gaps identified by the SCCM guideline panel $[5 \bullet \bullet$. They reflected that the assumptions of the guideline committee regarding their recommendations are not consistent with clinical practice. A clinician's belief in their ability to assess and effectively manage pain in critical illness often overrides written evidence-based guidance. Questions remain regarding the effectiveness of opioids to control pain when infused continuously, and the identification and prevention of withdrawal of opioids are not considered in the critically ill adults yet widely practiced in paediatric intensive care. High-quality trials investigating the effect of delirium assessment and the relationship between assessment and patient-centred outcomes, treatment decisions, and patient, family, and staff satisfaction are lacking. Evidence gaps need to be considered alongside improved methods for guideline implementation.

\section{Sedation Practice}

The 2018 Cochrane review update of "Protocol-directed sedation versus non-protocol-directed sedation in mechanically ventilated intensive care adults and children" included four studies with a total of 3323 participants (864 adults and 2459 paediatrics) [6•]. Three studies were single-centre, randomized control trials (RCTs) and one study was a multicentre cluster-RCT (see DESIST trial later). There was no clear evidence of benefit in duration of mechanical ventilation, mortality, or ICU length of stay using protocol-directed sedation. There was evidence of a reduction in hospital length of stay mean difference -3.09 days, $95 \%$ CI -5.08 to -1.10 ; moderate-quality evidence. In conclusion, future studies should account for differing contextual characteristics, with methodological strategies to reduce the risk of bias.

The ABCDEF bundle (assess, prevent, and manage pain; both spontaneous awakening and breathing trials: choice of analgesia and sedation; delirium assess, prevent, and manage; early mobility and exercise; family engagement/empowerment) aims to promote practice where patients are more awake, cognitively engaged, and physically active. The ICU Liberation Collaborative funded by the Gordon and Betty
Moore Foundation undertook a US quality improvement project to implement this bundle [7]. They included 15,000 patients from 68 ICUs covering 29 states and Puerto Rico, and the bundle was implemented with inconsistent and variable success. Patients who did receive more of the ABCDEF bundle elements each day had improved likelihood of surviving, less coma, delirium, and physical restraint, shorter ventilator time, fewer ICU readmissions, and were more likely to be discharged home. The low overall $8 \%$ total bundle performance meant that all seven components of ABCDEF only occurred 1 in 10 patient days.

The DESIST trial was undertaken in eight adult ICUs in Scotland and aimed to assess the effectiveness of three interventions to improve sedation practice [8]. These were education, regular feedback of ongoing sedation-analgesia quality data and a novel sedation-monitoring technology (the Responsiveness Index, Ri) based on facial electromyography, providing an alert for possible deep sedation. This cluster RCT used four combinations of the intervention from online education package in two units, up to the last two units implementing all three interventions. They subsequently published the results of a qualitative study to identify the challenges and barriers to implementation and engagement [9•].

An impressive 74 to $100 \%$ of nurses completed the online education. While education alone did not improve sedationanalgesia quality, it was associated with an almost $50 \%$ reduction in sedation-related adverse event rates (most commonly nasogastric tube removal). The Ri monitor attached to the patient alerted the team to the possibility of deep sedation with a green, amber, or red number (Ri) displayed. The Ri was red in $59 \%$ patients when attached and remained red for a median of $35 \%$ (IQR 18-65\%) monitored time. Nurses reported the Ri as a useful prompt to review sedation, but views were mixed about its usefulness, validity, impact on practice and intrusiveness.

Use of the monitor increased optimal sedation-analgesia quality by $7 \%$. Regular feedback of unit sedation quality delivered made no difference due to lack of intra-unit dissemination, it was thought to lack relevance to daily bedside practice, and often disbelieved. Predictive modelling concluded that a combination of education and responsiveness monitoring would result in a $10-11 \%$ improvement in proportion of shifts with optimal sedation without an increase in sedation-related adverse events. The qualitative data suggested that effects are partly explained by differences in engagement with interventions between ICUs. The takeaway messages are that a prompt is useful to review deep sedation, education is good, and reports about how well or badly an ICU performs does not lead to change.

\section{BIS}

Bispectral index (BIS) monitors, based on the processing of electroencephalographic signals, have reported benefits in the 
operating theatre and may overcome the restraints of sedation scales during deep sedation or during paralysis. A Cochrane review by Shetty and colleagues in 2018 concluded that there was insufficient evidence of benefit of BIS for ICU sedation, because of limited number of studies with evidence low to very low certainty [10]. More research is needed to monitor the brain in patients paralysed and deeply sedated, to ideally avoid burst suppression [11].

\section{No Sedation}

Given that sedation may worsen outcomes, a multi-national RCT (NONSEDA) was undertaken to determine if a plan of no sedation in patients requiring mechanical ventilation would result in an improved survival outcome over light sedation with daily sedation interruption [12••]. This was a follow on trial to a 2010 single centre study that showed benefit [13]. Eight centres across Denmark (5), Norway (2), and Sweden (1) randomized 710 patients to either no sedative infusions with morphine boluses as required or sedative infusions to maintain a level of RASS -2 to -3 (Fig. 1) [14]. Propofol was used infused for $48 \mathrm{~h}$ and then replaced by midazolam with daily sedation interruptions.

In the sedation group, the mean RASS score was -2.3 on day 1 increasing to -1.8 by day 7 , and in the non-sedation group the mean RASS score was -1.3 on day 1 and -0.8 on day 7 . In the no sedation group, $38 \%$ of patients received rescue sedation at some time during their stay. There was no difference in 90-day mortality between the two groups. There was 1 more day free from coma or delirium with no sedation versus light sedation and fewer thromboembolic events in the no sedation group at one patient $0.3 \%$ vs ten $(2.8 \%)$. This study is impressive in the maintenance of light sedation in the control (sedation) group. Compare this to SPICE 3 trial (discussed below) reporting RASS scores -3 to -5 in $45.6 \%$ of the control group throughout the first 2 days [15]. In NONSEDA, a follow-up survey of relative's satisfaction with
$39(73 \%)$ responses reported no differences between the groups with regard to relative's personal reactions or satisfaction with care, treatment or communication.

\section{Patient Experience}

A meta-synthesis and meta-summary to understand patients' experiences reported in qualitative studies of adult ICU patients receiving mechanical ventilation included nine studies published between 2015 and 2019, predominantly from Scandinavian countries, and 175 patients reported their experiences [16•]. The studies were mostly based on phenomenological-hermeneutical approaches, and two were mixed-method studies. Critically ill patients overall experience a sense of vulnerability describing (a) intense stress on body systems, (b) negative emotional situations, (c) feelings of being cared for in an ICU, and (d) support from family and loved ones. The conclusion was "At the unit and at the policy levels, strategies aimed at promoting family access to patients, maximizing the time available for families to be with patient and encouraging interactions are recommended (e.g., holding the patient's hand). Moreover an appropriate nurse-to-patientratio capable of ensuring presence at the bedside is strongly suggested".

\section{Alpha Agonists}

\section{Clonidine}

There is a paucity of evidence to support the use of clonidine because of a lack of controlled trials in critically ill adults. It is in common use in the UK; a 2014 survey published in 2016 ( $91 \%$ response rate) reported very frequent/frequent use of clonidine in $32.7 \%$ of units [17]. In Sweden, a survey reported that clinicians regularly prescribe clonidine, the indications

\section{RASS assessment Looks like this}

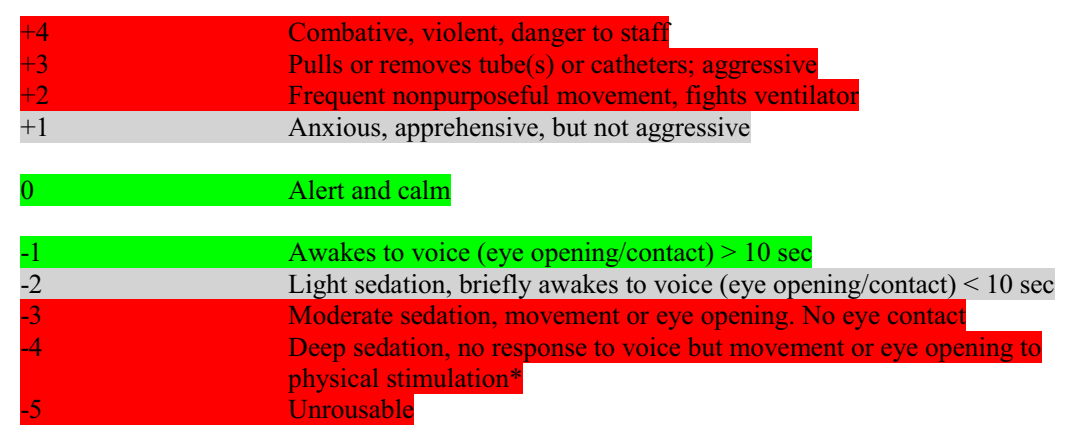

Fig. 1 Richmond Agitation Sedation Scale. The Richmond AgitationSedation Scale, RASS, differentiates response to verbal stimulus from that to a physical stimulus, unlike other sedation scores such as the Ramsay. *Physical stimulation: shaking and/or rubbing sternum.
Adapted from Sessler CN et al. The Richmond Agitation-Sedation Scale: validity and reliability in adult intensive care unit patients. American Journal of Respiratory and Critical Care Medicine 2002; 166: $1338-44$ 
being light sedation (32\%), non-invasive ventilation (23\%), and nightly sedation (18\%) [18].

A systematic review of studies until March 2016 in mechanically ventilated patients included eight RCTs, four in adults [19]. Six trials used intravenous clonidine with variation in dosing from 0.88 to $3 \mu \mathrm{g} / \mathrm{kg} / \mathrm{h}$. One trial reported clonidine as sole sedative, and two were postoperative patients with short periods of ventilation. The authors found no difference in duration of mechanical ventilation, mortality, or length of ICU stay and a high degree of clinical heterogeneity. They confirmed that clonidine reduces the total dose of opioids, while associated with increased incidence of clinically significant hypotension (RR $3.11,95 \% \mathrm{CI}=1.64$ to $5.87, \mathrm{I} 2=0 \%$, moderate certainty). There were three trials ongoing at the time of which two were terminated early due to difficulty recruiting patients, NCT01139996 and NCT02509273.

Cloesmeijer and colleagues developed the first population pharmacokinetic model for clonidine dosing in an adult ICU [20•]. They defined an optimal plasma concentration for ICU sedation as ranging from 1.5 to $4.0 \mu \mathrm{g} / \mathrm{L}$. Using different daily doses of intravenous clonidine in addition to standard sedation, they determined $1200 \mu \mathrm{g}$ per day which provided a target sedation concentration of more than $1.5 \mu \mathrm{g} / \mathrm{L}$. Rather than a loading dose, they recommend a doubling of the infusion rate for $6 \mathrm{~h}$ as more effective, reducing time to achieve steady-state to $5 \mathrm{~h}$ without peaks in plasma concentration.

\section{Dexmedetomidine}

A disproportionate number of dexmedetomidine-related systematic reviews and meta-analyses in critically ill ventilated patients of variable quality were published covering different patient populations and outcomes.

Two systematic reviews focused on patients difficult to wean from mechanical ventilation used the same six trials $[21,22]$. One described the evidence to suggest the use of dexmedetomidine being of low quality, whereas the other confidently concluded that dexmedetomidine was associated with a significant reduction in the time to extubation and shorter ICU stays. Two more reviews in patients with sepsis agreed that dexmedetomidine improved the short-term mortality of patients, a finding not upheld by subsequent clinical trials $[23,24]$. Four reviews in cardiac surgical patients generally agreed that dexmedetomidine use is likely to reduce delirium and lengths of stay, but with increased rates of bradycardia [25-28]. The two reviews in the neuro-critical care population concluded dexmedetomidine use appeared to be safe reviewing limited data $[29,30]$. Both reviews included cohort studies, generally thought not to be robust methodology.

Ng and colleagues' 2019 systematic review and metaanalysis of the effect of dexmedetomidine in ICU on agitation and delirium was in response to the publication of new studies
[31]. Twenty of the 25 RCTs included were in patients admitted to surgical ICUs. In eight RCTs, six in postoperative patients, delirium incidence was reduced with an odds ratio 0.36 $(0.26-0.51)$, bradycardia was more than twice as likely with dexmedetomidine, and hypotension was increased (odds ratio 1.89).

The UK National Institute for Health Care Research funded a comprehensive systematic review into the use of alpha agonists for sedation [32]. The authors used eighteen trials with a total of 2489 adult patients. They assessed that overall risk of bias as high or unclear particularly with relation to blinded outcome assessors. The length of ICU stay (mean difference -1.26 days, $95 \% \mathrm{CI}-1.96$ to -0.55 days, $\mathrm{I} 2=31 \%$ ) and time to extubation (mean difference -1.85 days, $95 \% \mathrm{CI}-2.61$ to 1.09 days, I $2=0 \%$ ) were significantly shorter among patients who received dexmedetomidine. Dexmedetomidine use was associated with a higher risk of bradycardia (RR 1.88, 95\% CI 1.28 to 2.77 , I $2=46 \%$.

The use of dexmedetomidine for non-invasive ventilation was studied in a systematic review/meta-analysis of trials published up until July 31, 2020 [33•]. Twelve studies were included with a total of 738 participants. Compared to any sedation strategy or placebo, dexmedetomidine reduced the risk of delirium (absolute risk reduction 16\%) and need for intubation and mechanical ventilation (absolute risk reduction 16\%). Any benefits of dexmedetomidine should be weighed against the probable undesirable effects of hypotension and bradycardia as use of dexmedetomidine was associated with an increased risk of bradycardia (RR 2.80, 95\% CI 1.92 to 4.07 , moderate certainty) and hypotension (RR $1.98,95 \%$ CI 1.32 to 2.98 , moderate certainty). This review was methodologically rigorous; however, due to the small number of studies pooled for each outcome, they were unable to examine funnel plots to detect publication bias.

\section{Dexmedetomidine and Light Sedation}

Early deep sedation is an independent predictor of time to extubation, hospital death, and 180-day mortality. The SPICE 3 trial was a multinational, open-label, randomized trial to investigate the effect of using dexmedetomidine as the primary and, if possible, sole agent for early light sedation [15]. The primary outcome was 90-day rate of death from any cause. Seventy-four ICUs in eight countries randomly assigned 4000 patients to receive either dexmedetomidine or usual care. The sedation goal was a RASS score of -2 (lightly sedated) to +1 (restless), unless the treating clinician decided there was an indication for deep sedation. The final analysis included 3904 patients and rates of death were similar in both groups at 90 days.

Clinicians deemed that on the first day around $60 \%$ of patients required sedation deeper than RASS -2 , i.e. briefly awakens to voice. In the first 2 days after randomization, the 
percentage of RASS scores in the target range of light sedation $(-2$ to +1$)$ was only $56.6 \%$ in the dexmedetomidine group and $51.8 \%$ in the usual-care group. The editorial noted that regardless of guidelines on the benefits of light sedation and wellconducted trials demonstrating the risks of deep sedation, beliefs drive practice and are slow to change [34]. Overall patients in this study were maintained at deeper levels of sedation by clinicians.

There was heterogeneity with respect to mortality rates below and above the median age (63.7 years) with higher mortality in younger patients (risk difference 4.4 (95\% confidence intervals 0.8 to 7.9 ) in the dexmedetomidine arm. The significance of the difference could not be determined and further analysis is awaited.

\section{Dexmedetomidine and Sepsis}

A subgroup analysis of the 2010 MENDS trial reported that patients with sepsis treated with dexmedetomidine as opposed to lorazepam had an improved mortality leading to an innovative trial undertaken in an Italian University hospital [35, 36•]. It aimed to determine if switching from propofol to dexmedetomidine reduced noradrenaline requirements in patients with septic shock. Thirty-eight mechanically ventilated patients requiring deep sedation RASS $-3 /-4$ were stabilized with a mean arterial pressure $65-75$ using noradrenaline while sedated with propofol and remifentanil. After $60 \mathrm{~min}$, the propofol infusion was replaced by dexmedetomidine, and then after $4 \mathrm{~h}$, the sedation infusions were reversed again. Noradrenaline requirements decreased with dexmedetomidine from $0.69 \pm 0.72 \mathrm{micrograms} / \mathrm{kg} / \mathrm{min}$ to $0.3 \pm 0.25$ micrograms $/ \mathrm{kg} / \mathrm{min}$, and increased again to $0.42 \pm 0.36 \mathrm{micro-}$ grams $/ \mathrm{kg} / \mathrm{min}$ post-dexmedetomidine. This supports experimental work and may be partly but not fully explained by the avoidance of propofol-related cardiovascular effects pronounced in septic shock.

The multi-centre DESIRE trial in eight Japanese ICUs published in JAMA randomized 201 consecutive patients admitted with sepsis requiring respiratory support to sedation with or without open-label dexmedetomidine [37]. (Curiously, they targeted a RASS of 0 during the day but -2 at night). The cumulative incidence of death at 28 days was $22.8 \%(n=19)$ in the dexmedetomidine group and $30.8 \%(n=28)$ in the control group $(P=.20)$. Although the difference in 28-day mortality was $8 \%$, it was powered for a $20 \%$ difference so may have been underpowered; however, the MENDS2 trial results suggest otherwise.

MENDS2 following on from MENDS was a multicentre trial comparing dexmedetomidine with propofol for sedation in mechanically ventilated patients with sepsis [38]. They recruited 432 patients to blinded propofol or dexmedetomidine. The primary outcome was number of delirium-free, coma-free days in the 14 days following randomization, and with the
RASS target that was set by the clinicians. There was no difference between groups in the primary outcome or any secondary outcomes. The patients had high severity of illness (APACHE II score median 27) and high 90-day mortality rate (38-39\%). Over $40 \%$ of patients needed supplementary midazolam and antipsychotics. Notably the dexmedetomidine dose given was relatively low, median $0.27 \mu \mathrm{g} / \mathrm{kg} / \mathrm{h}$ as compared with usual dose $0.2-1.4 \mu \mathrm{g} / \mathrm{kg} / \mathrm{h}$.

\section{Dexmedetomidine and Delirium}

The DahLIA study, "Dexmedetomidine to Lessen ICU Agitation", was a multi-centre double-blind, placebo-controlled, parallel-group RCT in mechanically ventilated patients who became so agitated that sedation could not be safely weaned for extubation [39]. Data was collected on 71 patients, 39 who received a dexmedetomidine infusion and 32 received a saline infusion for a sedation target of RASS 0 . The primary outcome was number of ventilatorfree hours up until day seven. The planned recruitment was 96 patients to detect a 20 -h difference; however, the trial was terminated early due to lack of time and funding. There was a significant difference in ventilator-free hours at 7 days, median $144.8 \mathrm{~h}$ vs $127.5 \mathrm{~h}$, with no difference in number of tracheostomies. The median time to extubation was $21.9 \mathrm{~h}$ for the dexmedetomidine vs $44.3 \mathrm{~h}$ for placebo, and median length of ICU stay is 2 days shorter. Delirium resolved more rapidly in patients who received dexmedetomidine $23.3 \mathrm{~h}$ vs. $40.0 \mathrm{~h}$, with a median of two additional delirium-free days during their ICU stay. Adverse events were rare. The authors did note that 21500 patients were screened to recruit 74 patients. These results regardless support the use of dexmedetomidine to manage symptoms of persistent agitation.

A two-centre delirium prevention study aimed to determine if a low dose nocturnal dexmedetomidine infusion prevented delirium and improved sleep in ICU adult patients [40•]. The hypothesis was that dexmedetomidine, by acting on the locus coeruleus, promotes natural sleep. One hundred patients were randomized in this phase 2 , blinded, placebo-controlled trial. All patient's sedative infusions were halved at $9.30 \mathrm{pm}$ and dexmedetomidine or dextrose $5 \%$ infusions started then discontinued at $6.30 \mathrm{am}$. A significant number of patients who received dexmedetomidine remained delirium-free during their admission, 40 of 50 patients versus 27 of 50 patients who received placebo. The total number of night-time hours spent at target goal of RASS -1 was greater in the dexmedetomidine group, $55 \%$ vs. $24 \%$. This study was unable to undertake a polysomography study relying on self-reported sleep quality assessments in 64 patients, which showed no difference between the two groups. 


\section{Dexmedetomidine and Sleep}

One study investigated the effect of enteral dexmedetomidine on sleep polysomnography in 15 participants [41]. Following $700 \mu \mathrm{g}$ of oral dexmedetomidine, the duration of non-rapid eye movement (non-REM) stage 2 sleep was increased by 63 (95\% CI, 19 to 107$) \min (P=0.010)$ and the duration of rapid eye movement (REM) sleep was decreased by 42 ( 5 to $78) \min (P=0.031)$. It supports the evidence that dexmedetomidine, similar to benzodiazepines, increases total sleep time but not stage 3 and REM sleep time.

\section{Other Drugs and Routes}

The N-methyl-d-aspartate receptor antagonist ketamine has also been shown to have analgesic properties, along with bronchodilatory effects and cardiovascular stimulation. Ketamine sedation in mechanically ventilated patients was reported in a systematic review and meta-analysis that included fifteen studies totalling 892 patients, non-randomized trials (12) included [42]. Every study was published since 2014, except one. As is often the case, reviewers highlighted the lack of data, or any demonstration of clinical benefit in the included studies.

One placebo-controlled, double-blind trial to determine if low dose ketamine decreased opioid consumption and delirium, albeit undertaken in 2011 to 2012, was published in 2018 in a surprisingly low-impact journal [43]. One hundred sixtytwo patients were randomized to a continuous infusion of lowdose ketamine $0.2 \mathrm{mg} / \mathrm{kg} / \mathrm{h}$ or equivalent volume of saline. The incidence of delirium was $21 \%$ (17) in the ketamine group and $37 \%$ (30) in the placebo group, a post hoc analysis reported a significant interaction between the ketamine bolus used for intubation in the ketamine group only with $16.6 \%$ delirium incidence if ketamine used for intubation as opposed to $26.3 \%$ if other drugs were used. There was no difference in opioid consumption between groups.

A systematic review and meta-analysis reviewed the literature on safety and efficacy of volatile anaesthetic agents in mechanically ventilated critically ill patients, as compared with intravenous propofol or midazolam [44•]. There were eight trials with 523 patients comparing volatile agents with midazolam or propofol reporting a reduction in extubation times using volatile agents (difference in means, $-52.7 \mathrm{~min}$; $95 \%$ confidence interval [CI], -75.1 to -30.3 ). Unsurprisingly, the difference was greater when volatile agents were compared with midazolam rather than propofol with a difference in means of $-292.2 \mathrm{~min}$ as opposed to -29.1 min. Their funnel plot revealed a large publication bias with trials reporting predominantly positive results. The findings suggested that the use of volatile agent sedation might be useful in postoperative patients requiring short-term ventilation, and a well-conducted research trial is required. Alternative methods of sedative delivery may be needed in the not too distant future [45].

A multi-centre, randomized controlled trial compared enteral versus intravenous sedation [46]. Three hundred and forty-eight patients from 12 Italian ICUs were randomized to receive either midazolam or propofol infused, or hydroxyzine, lorazepam, and melatonin enterally for sedation. This was a superiority trial. There was no difference in the primary outcome of percentage of work shifts in which patients reached a target or RASS $0 \pm 1$. Half of the patients in the enteral sedation group had protocol violations so the groups were not adequately separated. Interestingly there were more unplanned extubations in the enteral sedation group but did not need reintubation, and the enteral sedation group received more enteral nutrition.

\section{COVID-19 and Sedation}

The SARS-coronavirus or COVID-19 pandemic has presented new challenges to clinicians aiming for optimal sedation in mechanically ventilated patients [47]. Hospitals have been overwhelmed with critically ill patients generally resulting a lower skilled nurse to patient ratio. More patients require longer periods of ventilation, use of neuromuscular drugs, and proning with deep sedation. There are reported shortages of commonly used sedative drugs.

A multinational, multi-centre cohort study involving 69 ICUs in 14 countries collected data on 2088 patients [48]. The median RASS score on ventilation was -4 ( -5 to -3$)$ and the median number of days in coma was ten (IQR 6 to 16). This compares with days in coma of 1 day (IQR 1-2) in the 2018 delirium treatment MIND-USA trial completed by the same investigator group [49]. Importantly in this cohort study, more than $50 \%$ of patients had agitation. Before the COVID-19 pandemic, in critically ill patients, the reported incidence of new agitated delirium was up to $13 \%$, with an overall prevalence of up to $20 \%$ [50]. Similarly, Helms and colleagues reviewed 58 consecutive ICU patients with COVID-19, of whom 40 (69\%) became agitated following cessation of muscle relaxation and sedation [51].

\section{Conclusion}

Clinical trials to date do not fully support the use of dexmedetomidine other than to manage agitation. Meantime, more attention is being paid to older drugs in common, e.g. clonidine and ketamine, and delirium risk factors and management. Knowledge translated into guidelines and protocols are all very well, but studies need to look at translating evidence into practice. 


\section{Compliance with Ethical Standards}

Conflict of Interest Neither of the authors has any potential conflicts of interest to disclose.

\section{References}

Papers of particular interest, published recently, have been highlighted as:

- Of importance

•- Of major importance

1. Devlin JW, Skrobik Y, Gélinas C, Needham DM, Slooter AJC, Pandharipande PP, et al. Clinical practice guidelines for the prevention and management of pain, agitation/sedation, delirium, immobility, and sleep disruption in adult patients in the ICU. Crit Care Med. 2018;46:e825-73. https://doi.org/10.1097/CCM. 0000000000003299 Comprehensive and in-depth account of the evidence available and basis on which decisions were made.

2. Celis-Rodríguez E, Díaz Cortés JC, Cárdenas Bolívar YR, Carrizosa González JA, Pinilla DI, Ferrer Záccaro LE, et al. Evidence-based clinical practice guidelines for the management of sedoanalgesia and delirium in critically ill adult patients. Med Intensiva. 2020;44:171-84. English, Spanish. https://doi.org/10. 1016/j.medin.2019.07.013.

3. Martin J, Heymann A, Bäsell K, Baron R, Biniek R, Bürkle H, et al. Evidence and consensus-based German guidelines for the management of analgesia, sedation and delirium in intensive care-short version. Ger Med Sci. 2010;8:Doc02. https://doi.org/10.3205/ 000091.

4. Whitehouse T, Snelson C, Grounds M. Intensive care society review of best practice for analgesia and sedation in the critical care: Intensive Care Society UK; 2014.

5.• Pisani MA, Devlin JW, Skrobik Y. Pain and delirium in critical illness: an exploration of key 2018 SCCM PADIS guideline evidence gaps. Semin Respir Crit Care Med. 2019;40(5):604-13. https://doi.org/10.1055/s-0039-1698809 Describes sedation practice in context and how it applies to the PADIS guidelines. Discusses what is assumed, what is missing and what is needed to fill the gaps.

6. Aitken LM, Bucknall T, Kent B, Mitchell M, Burmeister E, Keogh SJ. Protocol-directed sedation versus non-protocol-directed sedation in mechanically ventilated intensive care adults and children. Cochrane Database Syst Rev. 2018;11(11):CD009771. https://doi. org/10.1002/14651858.CD009771.pub3 Shows how the evidence is not always there to support recommendations.

7. Pun BT, Balas MC, Barnes-Daly MA, Thompson JL, Aldrich JM, Barr J, et al. Caring for critically ill patients with the $\mathrm{ABCDEF}$ bundle: results of the ICU liberation collaborative in over 15,000 adults. Crit Care Med. 2019;47(1):3-14. https://doi.org/10.1097/ CCM.0000000000003482.

8. Walsh TS, Kydonaki K, Antonelli J, Stephen J, Lee RJ, Everingham K, et al. Development and Evaluation of Strategies to Improve Sedation Practice in Intensive Care (DESIST) study investigators. Staff education, regular sedation and analgesia quality feedback, and a sedation monitoring technology for improving sedation and analgesia quality for critically ill, mechanically ventilated patients: a cluster randomised trial. Lancet Respir Med. 2016;4(10):807-17. https://doi.org/10.1016/S2213-2600(16) 30178-3.
9. Kydonaki K, Hanley J, Huby G, Antonelli J, Walsh TS. Development and Evaluation of Strategies to Improve Sedation practice in inTensive care (DESIST) study investigators. Challenges and barriers to optimising sedation in intensive care: a qualitative study in eight Scottish intensive care units. BMJ Open. 2019;9(5):e024549. https://doi.org/10.1136/bmjopen-2018024549 Provides an insight into bedside clinicians thought processes when prescribing or delivering sedation to patients.

10. Shetty RM, Bellini A, Wijayatilake DS, Hamilton MA, Jain R, Karanth S, et al. BIS monitoring versus clinical assessment for sedation in mechanically ventilated adults in the intensive care unit and its impact on clinical outcomes and resource utilization. Cochrane Database Syst Rev. 2018;2(2):CD011240. https://doi. org/10.1002/14651858.CD011240.pub2.

11. Hogan J, Sun H, Aboul Nour H, Jing J, Tabaeizadeh M, Shoukat M, et al. Burst suppression: causes and effects on mortality in critical illness. Neurocrit Care. 2020;33(2):565-74. https://doi.org/10. 1007/s12028-020-00932-4.

12.• Olsen HT, Nedergaard HK, Strøm T, Oxlund J, Wian KA, Ytrebø LM, et al. Nonsedation or light sedation in critically ill, mechanically ventilated patients. N Engl J Med. 2020;382(12):1103-11. https://doi.org/10.1056/NEJMoa1906759 Seminal study into what can be achieved and consider the no effect may be likely due to excellent sedation practice in the control group.

13. Strøm T, Martinussen T, Toft P. A protocol of no sedation for critically ill patients receiving mechanical ventilation: a randomised trial. Lancet. 2010;375(9713):475-80. https://doi.org/10.1016/ S0140-6736(09)62072-9.

14. Sessler CN, Gosnell MS, Grap MJ, Brophy GM, O'Neal PV, Keane KA, et al. The Richmond Agitation-Sedation Scale: validity and reliability in adult intensive care unit patients. Am J Respir Crit Care Med. 2002;166(10):1338-44. https://doi.org/10.1164/rccm. 2107138.

15. Shehabi Y, Howe BD, Bellomo R, Arabi YM, Bailey M, Bass FE, et al. ANZICS Clinical Trials Group and the SPICE III Investigators. Early sedation with dexmedetomidine in critically ill patients. N Engl J Med. 2019;380(26):2506-17. https://doi.org/ 10.1056/NEJMoa1904710.

16. Danielis M, Povoli A, Mattiussi E, Palese A. Understanding patients' experiences of being mechanically ventilated in the intensive care unit: findings from a meta-synthesis and meta-summary. J Clin Nurs. 2020;29(13-14):2107-24. https://doi.org/10.1111/jocn. 15259 Provides an insight into the patient experiences.

17. Richards-Belle A, Canter RR, Power GS, Robinson EJ, Reschreiter $\mathrm{H}$, Wunsch $\mathrm{H}$, et al. National survey and point prevalence study of sedation practice in UK critical care. Crit Care. 2016;20(1):355.

18. Talsi O, Kiiski Berggren R, Johansson G, Winsö O. A national survey on routines regarding sedation in Swedish intensive care units. Ups J Med Sci. 2019;124(3):199-202.

19. Wang JG, Belley-Coté E, Burry L, Duffett M, Karachi T, Perri D, et al. Clonidine for sedation in the critically ill: a systematic review and meta-analysis. Crit Care. 2017;21(1):75. https://doi.org/10. 1186/s13054-017-1610-8.

20. Cloesmeijer ME, van den Oever HLA, Mathôt RAA, Zeeman M, Kruisdijk-Gerritsen A, Bles CMA, et al. Optimising the dose of clonidine to achieve sedation in intensive care unit patients with population pharmacokinetics. Br J Clin Pharmacol. 2020;86(8): 1620-31. https://doi.org/10.1111/bcp.14273 Excellent pharmacokinetic study in a drug commonly used with considerable variation in dosing. Very useful recommendation regarding doubling infusion for six hours rather than using a bolus loading does.

21. Dupuis S, Brindamour D, Karzon S, Frenette AJ, Charbonney E, Perreault MM, et al. A systematic review of interventions to facilitate extubation in patients difficult-to-wean due to delirium, agitation, or anxiety and a meta-analysis of the effect of 
dexmedetomidine. Can J Anaesth. 2019;66(3):318-27. English. https://doi.org/10.1007/s12630-018-01289-1.

22. Buckley MS, Smithburger PL, Wong A, Fraser GL, Reade MC, Klein-Fedyshin M, et al. Dexmedetomidine for facilitating mechanical ventilation extubation in difficult-to-wean ICU patients: systematic review and meta-analysis of clinical trials. J Intensive Care Med. Jul. 2020;6:885066620937673. https://doi.org/10. $1177 / 0885066620937673$

23. Chen P, Jiang J, Zhang Y, Li G, Qiu Z, Levy MM, et al. Effect of dexmedetomidine on duration of mechanical ventilation in septic patients: a systematic review and meta-analysis. BMC Pulm Med. 2020;20(1):42. https://doi.org/10.1186/s12890-020-1065-6.

24. Zamani MM, Keshavarz-Fathi M, Fakhri-Bafghi MS, HirbodMobarakeh A, Rezaei N, Bahrami A, et al. Survival benefits of dexmedetomidine used for sedating septic patients in intensive care setting: a systematic review. J Crit Care. 2016;32:93-100. https:// doi.org/10.1016/j.jcrc.2015.11.013 Epub 2015 Nov 23.

25. Liu X, Xie G, Zhang K, Song S, Song F, Jin Y, et al. Dexmedetomidine vs propofol sedation reduces delirium in patients after cardiac surgery: a meta-analysis with trial sequential analysis of randomized controlled trials. J Crit Care. 2017;38: 190-6. https://doi.org/10.1016/j.jcrc.2016.10.026.

26. Nguyen J, Nacpil N. Effectiveness of dexmedetomidine versus propofol on extubation times, length of stay and mortality rates in adult cardiac surgery patients: a systematic review and meta-analysis. JBI Database System Rev Implement Rep. 2018;16(5):1220 39. https://doi.org/10.11124/JBISRIR-2017-003488.

27. Wang G, Niu J, Li Z, Lv H, Cai H. The efficacy and safety of dexmedetomidine in cardiac surgery patients: a systematic review and meta-analysis. PLoS One. 2018;13(9):e0202620. https://doi. org/10.1371/journal.pone.0202620.

28. Wu M, Liang Y, Dai Z, Wang S. Perioperative dexmedetomidine reduces delirium after cardiac surgery: a meta-analysis of randomized controlled trials. J Clin Anesth. 2018;50:33-42. https://doi.org/ 10.1016/j.jclinane.2018.06.045 Epub 2018 Jun 27.

29. Tsaousi GG, Lamperti M, Bilotta F. Role of dexmedetomidine for sedation in neurocritical care patients: a qualitative systematic review and meta-analysis of current evidence. Clin Neuropharmacol. 2016;39(3):144-51. https://doi.org/10.1097/WNF. 0000000000000151 .

30. Tran A, Blinder H, Hutton B, English SW. A systematic review of alpha-2 agonists for sedation in mechanically ventilated neurocritical care patients. Neurocrit Care. 2018;28(1):12-25. https://doi.org/10.1007/s12028-017-0388-5.

31. Ng KT, Shubash CJ, Chong JS. The effect of dexmedetomidine on delirium and agitation in patients in intensive care: systematic review and meta-analysis with trial sequential analysis. Anaesthesia. 2019;74(3):380-92. https://doi.org/10.1111/anae.14472.

32. Cruickshank M, Henderson L, MacLennan G, Fraser C, Campbell M, Blackwood B, et al. Alpha-2 agonists for sedation of mechanically ventilated adults in intensive care units: a systematic review. Health Technol Assess. 2016;20(25):v-xx, 1-117. https://doi.org/ 10.3310/hta20250.

33. Lewis K, Piticaru J, Chaudhuri D, Basmaji J, Fan E, Møller MH, et al. Safety and efficacy of dexmedetomidine in acutely ill adults requiring non-invasive ventilation: a systematic review and metaanalysis of randomized trials. Chest. 2021:S0012-3692(21)000325. https://doi.org/10.1016/j.chest.2020.12.052 Well-conducted systematic review to support the use of dexmedetomidine in a key patient population that often present a challenge to critical care clinicians. Well balanced discussion puts the findings in context.

34. Coursin DB, Skrobik Y. What Is Safe Sedation in the ICU? N Engl J Med. 2019;380(26):2577-8. https://doi.org/10.1056/ NEJMe1906522.
35. Pandharipande PP, Sanders RD, Girard TD, McGrane S, Thompson JL, Shintani AK, et al. Effect of dexmedetomidine versus lorazepam on outcome in patients with sepsis: an a prioridesigned analysis of the MENDS randomized controlled trial. Crit Care. 2010;14(2):R38. https://doi.org/10.1186/cc8916 Erratum in: Crit Care. 2011;15(1):402. PMID: 20233428; PMCID: PMC2887145.

36. Morelli A, Sanfilippo F, Arnemann P, Hessler M, Kampmeier TG, D'Egidio A, et al. The effect of propofol and dexmedetomidine sedation on norepinephrine requirements in septic shock patients: a crossover trial. Crit Care Med. 2019;47(2):e89-95. https://doi. org/10.1097/CCM.0000000000003520 Interesting trial with participants acting as their own controls that seems to show dexmedetomidine reduces vasopressor requirements.

37. Kawazoe Y, Miyamoto K, Morimoto T, Yamamoto T, Fuke A, Hashimoto A, et al. Dexmedetomidine for Sepsis in Intensive Care Unit Randomized Evaluation (DESIRE) Trial Investigators. effect of dexmedetomidine on mortality and ventilator-free days in patients requiring mechanical ventilation with sepsis: a randomized clinical trial. JAMA. 2017;317(13):1321-8. https://doi.org/10. 1001/jama.2017.2088.

38. Hughes CG, Mailloux PT, Devlin JW, Swan JT, Sanders RD, Anzueto A, et al. MENDS2 Study Investigators. Dexmedetomidine or propofol for sedation in mechanically ventilated adults with sepsis. N Engl J Med. 2021. https://doi.org/10. 1056/NEJMoa2024922 Epub ahead of print.

39. Reade MC, Eastwood GM, Bellomo R, Bailey M, Bersten A, Cheung B, et al. Effect of dexmedetomidine added to standard care on ventilator-free time in patients with agitated delirium: a randomized clinical trial. JAMA. 2016;315(14):1460-8. https://doi.org/10. 1001/jama.2016.2707 Erratum in: JAMA. 2016 Aug 16;316(7): 775 .

40. Skrobik Y, Duprey MS, Hill NS, Devlin JW. Low-dose nocturnal dexmedetomidine prevents ICU delirium. a randomized, placebocontrolled trial. Am J Respir Crit Care Med. 2018;197(9):1147-56. https://doi.org/10.1164/rccm.201710-1995OC Complicated trial design generated from hypothesis relating to improved sleep (not proven) shows overnight dexmedetomidine reduces delirium.

41. Chamadia S, Hobbs L, Marota S, Ibala R, Hahm E, Gitlin J, et al. Oral dexmedetomidine promotes non-rapid eye movement stage 2 sleep in humans. Anesthesiology. 2020;133(6):1234-43. https:// doi.org/10.1097/ALN.0000000000003567.

42. Manasco AT, Stephens RJ, Yaeger LH, Roberts BW, Fuller BM. Ketamine sedation in mechanically ventilated patients: a systematic review and meta-analysis. J Crit Care. 2020;56:80-8. https://doi. org/10.1016/j.jcrc.2019.12.004.

43. Perbet S, Verdonk F, Godet T, Jabaudon M, Chartier C, Cayot S, et al. Low doses of ketamine reduce delirium but not opiate consumption in mechanically ventilated and sedated ICU patients: a randomised double-blind control trial. Anaesth Crit Care Pain Med. 2018;37(6):589-95. https://doi.org/10.1016/j.accpm.2018. 09.006 .

44. Jerath A, Panckhurst J, Parotto M, Lightfoot N, Wasowicz M, Ferguson ND, et al. Safety and efficacy of volatile anesthetic agents compared with standard intravenous midazolam/propofol sedation in ventilated critical care patients: a meta-analysis and systematic review of prospective trials. Anesth Analg. 2017;124(4):1190-9. https://doi.org/10.1213/ANE.0000000000001634 A good case made to consider volatile agents as sedatives at least in shortterm ventilated patients.

45. Jerath A, Ferguson ND, Cuthbertson B. Inhalational volatile-based sedation for COVID-19 pneumonia and ARDS. Intensive Care Med. 2020;46(8):1563-6. https://doi.org/10.1007/s00134-02006154-8 Epub 2020 Jun 25. 
46. Mistraletti G, Umbrello M, Salini S, Cadringher P, Formenti P, Chiumello D. Enteral versus intravenous approach for the sedation of critically ill patients: a randomized and controlled trial. Crit Care. 2019;23(1):3. https://doi.org/10.1186/s13054-018-2280-x.

47. Phua J, Weng L, Ling L, Egi M, Lim CM, Divatia JV, et al. Intensive care management of coronavirus disease 2019 (COVID19): challenges and recommendations. Lancet Respir Med. 2020;8(5):506-17. https://doi.org/10.1016/S2213-2600(20)301612 Erratum in: Lancet Respir Med. 2020 May;8(5):e42.

48. Pun BT, Badenes R, Heras La Calle G, Orun OM, Chen W, Raman $\mathrm{R}$, et al. Prevalence and risk factors for delirium in critically ill patients with COVID-19 (COVID-D): a multicentre cohort study. Lancet Respir Med. 2021;9(3):239-250. https://doi.org/10.1016/ S2213-2600(20)30552-X.

49. Girard TD, Exline MC, Carson SS, Hough CL, Rock P, Gong MN, et al. Haloperidol and ziprasidone for treatment of delirium in critical illness. N Engl J Med. 2018;379(26):2506-16. https://doi. org/10.1056/NEJMoa1808217.

50. Krewulak KD, Stelfox HT, Leigh JP, Ely EW, Fiest KM. Incidence and prevalence of delirium subtypes in an adult ICU: a systematic review and meta-analysis. Crit Care Med. 2018;46(12):2029-35. https://doi.org/10.1097/CCM.0000000000003402.

51. Helms J, Kremer S, Merdji H, Clere-Jehl R, Schenck M, Kummerlen C, et al. Neurologic features in severe SARS-CoV-2 infection. N Engl J Med. 2020;382(23):2268-70. https://doi.org/ 10.1056/NEJMc2008597 Epub 2020 Apr 15.

Publisher's Note Springer Nature remains neutral with regard to jurisdictional claims in published maps and institutional affiliations. 